\title{
Elementos conceptuales de la globalización para la inclusión del riesgo en la planificación regional
}

\author{
Oswaldo Mesías Rosas
}

Invitado internacional

Universidad Central de Venezuela, Venezuela

omesias@gmail.com

Recibido: 15 de diciembre del 2016

Aceptado: 9 de junio del 2017

\section{Oswaldo Mesías Rosas}

Arquitecto y magister en hábitat de la Universidad Nacional de Colombia, actual doctorando en urbanismo de la Universidad Central de Venezuela - UCV. Docente Auxiliar del programa de Arquitectura de la Universidad de Nariño, con sede en la ciudad de Pasto, al sur de Colombia.

\begin{abstract}
Resumen
El objetivo de este trabajo es presentar algunos conceptos fundamentales que deben ser considerados para presentar propuestas que ayuden a definir el uso y la organización de las áreas vulnerables frente a amenazas naturales, entendido en el actual contexto de globalización económica que influye fuertemente en la planificación territorial. Estos elementos contribuirán a fortalecer tanto el marco teórico como metodológico para aportar al desarrollo de políticas urbano-regionales que contribuyan a la gestión de riesgos. La hipótesis presentada para el desarrollo de este artículo argumenta que, para el actual fenómeno de globalización dominante, todavía podemos encontrar oportunidades en las que logremos superar el paradigma exclusivo que este modelo ha establecido a favor de una planificación territorial que sólo atiende a los intereses de la reproducción del capital. A partir de la lógica del desarrollo local y de una posición endógena, especialmente en lo que respecta a todo lo relacionado con la gestión del riesgo de origen natural, es posible plantear alternativas del ordenamiento territorial sostenibles que consideren las opiniones de las comunidades afectadas y que garanticen la preservación de la vida y los bienes de las personas que son directamente aquejadas.
\end{abstract}

Palabras clave: desarrollo regional; gestión del riesgo; globalización; planificación regional.

\section{Conceptual elements of globalization for risk management in land} planning

\begin{abstract}
This paper aims to provide some core concepts, to take into account, to present proposals that help to define the use and organization of vulnerable areas against natural threats, this understood in the current context of the economic globalization, which strongly influences the territory planning. These elements will help to strengthen both the theoretical framework and the methodological development to contribute developing urban-regional policies that contribute with risk management. The hypothesis presented for the development of this article, argues that for the current dominant globalization phenomenon, we still can find loopholes where we can overcome the exclusive paradigm this model has established in favor of a territory planning that only caters to the interests of capital reproduction. From the rationale of the local development and from an endogenous position, especially regarding everything related with natural source risk management, we can present sustainable land planning models that consider the views of the affected communities vouching for the preservation of life and assets of the people that are directly involved.
\end{abstract}

Keywords: regional development; risk management; globalization; land planning. 


\section{Introducción:}

ste ensayo tiene como objetivo presentar conceptos claves que nos plantea el actual modelo predominante de la globalización para tener en cuenta al tratar de plantear propuestas que pretendan definir el uso y la organización del territorio; elementos que servirán para fortalecer las propuestas pertinentes a la inclusión de la gestión del riesgo como una dimensión transversal en la planificación del territorio".

Para entender cómo se dan las condiciones en nuestra sociedad actual, es importante que nos adentremos en algunas precisiones conceptuales que nos permitan comprender cómo funcionan las políticas globales de donde se desprenden las políticas nacionales y regionales. Las políticas regionales, como políticas de Estado, se enmarcan dentro de las leyes generales de la economía mundial que nos rige. Todo país independiente de su orientación política, debe tener claro este marco en el que se desenvuelve y al cual no puede ignorar ni estar ajeno; esta situación se ve aún más comprometida para nuestros países latinoamericanos denominados como "países en desarrollo", que por su alto grado de dependencia se ven aún más ligados a los dictámenes de las políticas globales.

Figura 1. Globalización y planificación. Fuente: elaboración propia.

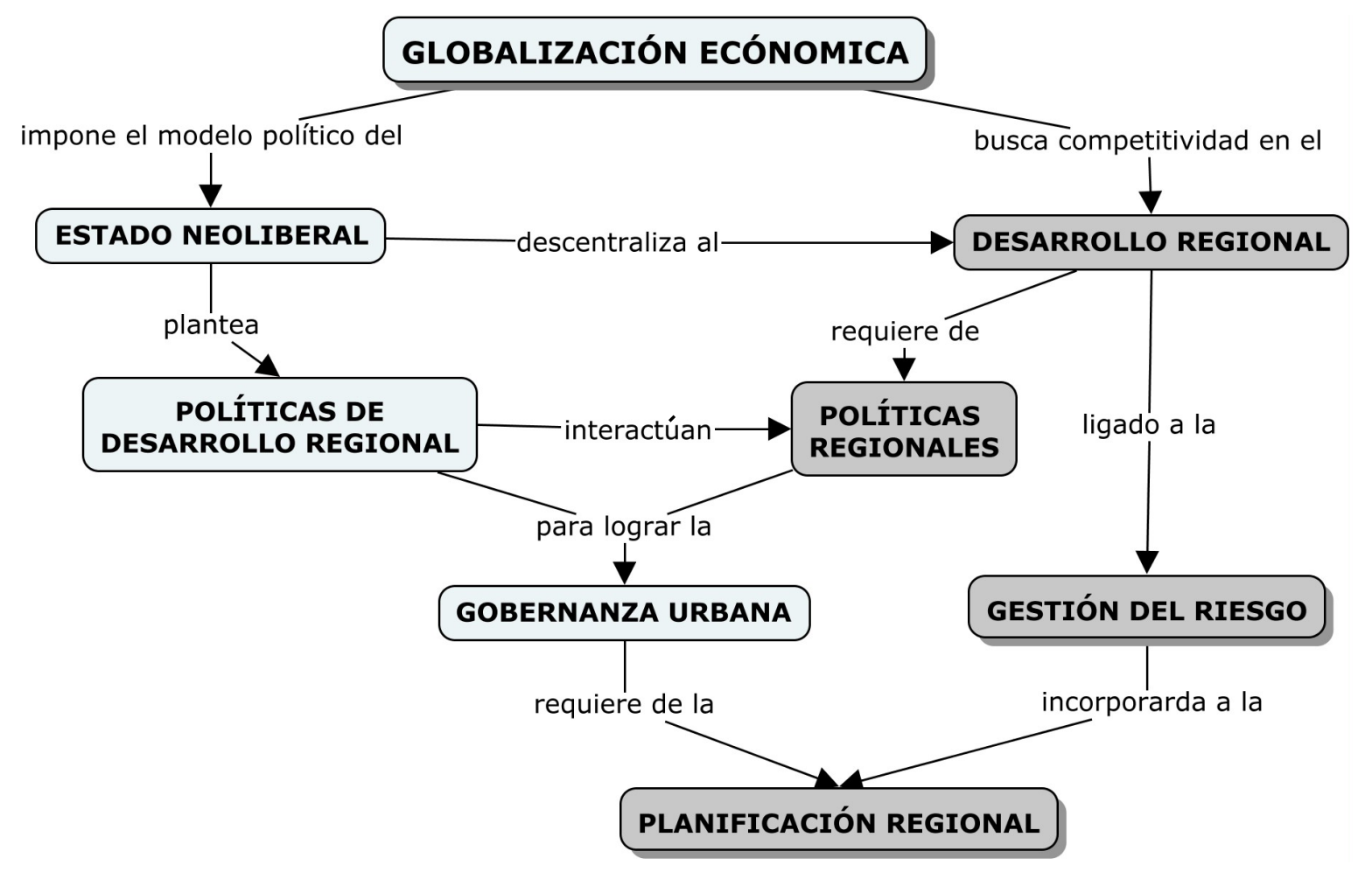

El texto, que se sintetiza en el mapa conceptual de la figura 1, se organiza en torno a seis apartados. Inicia desde entender el actual contexto de la globalización, así como las características y exigencias del Estado neoliberal, sus tensiones y contradicciones. Como temas relevantes, se aborda a continuación, la gobernanza urbana para acercarse a la planificación urbano regional, y los tipos de políticas regionales que implican. Seguidamente se hace énfasis en el reposicionamiento de las ciudades y las regiones en la economía global, donde se insiste en la importancia del desarrollo local. Se aborda, a continuación, la gestión del riesgo y globalización para relacionarla luego con el tema de la inclusión de la gestión del riesgo en la planificación territorial, lo que permite finalmente plantear conclusiones con una mirada alternativa que vaya desde lo local a lo global.

\section{El actual contexto de la globalización}

\section{El Estado neoliberal}

La globalización económica se fortalece después de la Segunda Guerra Mundial, lo que le permitió organizar el mundo a su imagen liberal, logró la reconstrucción de los países devastados, la construcción de la comunidad europea y de naciones, la restauración de la democracia y un orden económico basado en el libre comercio. Luego, tras la caída de la Unión Soviética, el Consenso de Washington, durante los años 80 y 90 , fue el acuerdo mundial encargado de implantar los tres pilares fundamentales del modelo
$1 \quad$ Este ensayo busca alimentar los marcos teórico y metodológico de la tesis en construcción en el doctorado en urbanismo titulada: "La gestión del riesgo volcánico en la planificación regional, estudio de caso volcán Galeras - Pasto, Colombia". 
político del neoliberalismo: la austeridad fiscal, la privatización y la liberalización de los mercados, con el apoyo de la políticas del neoliberalismo, basadas en la concepción fundamentalista de que los mercados se corrigen a sí mismos, asignan los recursos eficientemente y sirven bien al interés público.

La globalización como marco contextual en el que hoy nos encontramos, tiene tres grandes implicaciones. En el aspecto económico, la expansión dominante del sistema capitalista; en el aspecto político, hace perder hegemonía a los Estados como principal institución de poder político de gobierno y de administración; y en el aspecto cultural, con la expansión de las Tecnologías de la Información y de la Comunicación (TIC), terminan proponiendo también la homogeneización de la cultura a nivel global. "El neoliberalismo es, ante todo, una teoría de prácticas político-económicas que afirma que la mejor manera de promover el bienestar del ser humano, consiste en no restringir el libre desarrollo de las capacidades y de las libertades empresariales del individuo" (Harvey, 2007a, p. 8).

\section{Tensiones y contradicciones}

La neoliberalización, como proyecto político dominante se encuentra, en cierto modo, ligado a la restauración o a la reconstrucción del poder de las élites económicas. En la práctica es el modelo de una clase dominante, que encierra muchos bemoles que permiten afirmar que el Estado neoliberal también es una forma política inestable y contradictoria. "Treinta años de libertades neoliberales no sólo han servido para restaurar el poder a una clase capitalista definida en términos reducidos. También han generado inmensas concentraciones de poder corporativo en el campo de la energía, los medios de comunicación, la industria farmacéutica, el transporte, e incluso la venta al por menor" (Harvey, 2007a, p. 42).

En el seno de la teoría Neoliberal, pese a sus afirmaciones de integración y de equidad en las posibilidades, existen conflictos y contradicciones, que para autores como Boisier (2005), Borja y Castells (2004), De Mattos (2010), Harvey (2007a), Max-Neef (1994), Sen (2000) o Stiglitz (2002) lo ponen de manifiesto en los siguientes puntos:

- La competencia acaba convertida en monopolio o en oligopolio que termina expulsando a las empresas más débiles.

- Las fallas de mercado se producen cuando los individuos y las compañías eluden asumir la totalidad de los costes imputados a su actividad, como la contaminación y los riesgos que ésta genera.

Los agentes que actúan en el mercado no tienen igualdad de condiciones para acceso a la misma información, tan solo los jugadores más poderosos son los más informados, generando así relaciones de poder asimétricas que tienden a incrementarse.

El desarrollo tecnológico puede descontrolarse debido a que los sectores dedicados únicamente a la innovación tecnológica crean nuevos productos cuando todavía no existe mercado para ellos.

Como problema político fundamental se destaca un excesivo individualismo posesivo, en contradicción con un deseo de vida colectiva significativa de las comunidades locales.

El principal malestar, nos insiste Stiglitz (2002), fue que el problema no era de la globalización, sino cómo se gestionaba el proceso de la misma. En su implementación planetaria el proceso de globalización se caracteriza por ser antidemocrático y destructor de los recursos naturales, creador de riqueza para algunos y de mejora social para otros; pero a su vez, crea también pobreza y exclusión para muchos.

\section{Conflicto urbano y competencia urbana}

Dentro del contexto actual de la globalización económica y el modelo político del neoliberalismo, nuevas características de las transformaciones urbanas se manifiestan: las ciudades crecen en contextos globalizados y no nacionales, no es el Estado sino el mercado el que fija rumbo urbano, y la fascinación por la ciudad gira alrededor de su 
espectacularidad y no su materialidad. "Hoy en el entorno implacable de la globalización y la competitividad, la ciudad competitiva se ha convertido en una doctrina que domina el discurso oficial del desarrollo urbano en la última década." (Brand, 2009, p.10).

Asistimos al cambio de los sistemas de producción fordistas respaldados por el Estado de bienestar keynesiano, que tenía una política urbana de subsidios directos de apoyo al ciudadano en materia de vivienda, salud, educación, entre otros, al de un modelo de Estado neoliberal que da protagonismo al mercado. Se da un giro hacia la gobernanza empresarial, donde se subsidia a las empresas para que oferten como mercancías los servicios, antes asumidos directamente por el Estado. El cambio de la política urbana y el paso al empresarialismo han facilitado la transición a una forma de acumulación más flexible basada en el mercado y geográficamente mucho más abierta.

El resultado es un eclecticismo de consumo urbano demoledor del arraigo cultural y de la unidad territorial, pero contradictoriamente, la venta de la ciudad como ubicación para determinada actividad depende en gran medida de la creación de un imaginario urbano atractivo. El empresarialismo urbano se mezcla aquí con la búsqueda de una identidad local, donde las particularidades y las diferenciaciones del lugar se vuelven fundamentales para la gobernanza urbana.

Tenemos un cambio entre el Estado central y el local. Las actividades estatales de impacto local se liberan del Estado del bienestar, teniendo como consecuencia problemas sociales y económicos graves, que se expresan en el territorio a modo de ciudad dual, donde coexisten centros urbanos renovados y una periferia circundante de empobrecimiento creciente. Se desprende dentro de este contexto una crítica ácida al empresarialismo urbano, que si bien puede traer ciertos beneficios políticos y económicos, en el fondo no se centra en los problemas económicos y sociales del conjunto de su población.

\section{Disparidades regionales}

Generalmente, bajo las directrices del Estado neoliberal, el crecimiento económico de un país no se traduce en desarrollo que se distribuya de manera igual sobre su territorio nacional; siempre se presentan disparidades económicas entre las regiones. La disparidad regional o desigualdad regional hace referencia a la inequidad de bienestar 0 de desarrollo entre regiones. "No debe confundirse desigualdad entre personas y entre regiones, aunque ambas pueden estar ligadas. Es completamente posible que un país sufra de enormes desigualdades sociales (entre personas o familias), pero que estas se reproduzcan exactamente de la misma manera, en todas las regiones del país. En este caso habrá disparidad regional." (Polèse, 1998, p. 178). Lo que significa, que una política nacional que pretenda reducir las disparidades regionales, no necesariamente se traducirá en una mayor igualdad social. Es importante también no confundir dos tipos de desigualdades, la desigualdad en el nivel de bienestar relacionado con el ingreso familiar, con la desigualdad en la distribución espacial relacionado con la diferencia de concentración de actividades económicas y poblacionales sobre el territorio nacional.

Las disparidades regionales existirán siempre, mientras las diferencias en el espacio geográfico, las distancias, las condiciones socioculturales, las condiciones climáticas, las condiciones históricas, o cualquier otro factor, impida la integración completa de los mercados, y para la economía globalizada no hay nada que obligue al mercado a distribuirse equilibradamente. Tanto a nivel de países en el contexto internacional, como de regiones al interior del territorio nacional, siempre habrá ganadores y perdedores. Para el caso de las desigualdades sociales en los países de América Latina, asociadas también a desigualdades regionales, estas son más grandes que las de países desarrollados.

El concepto centro-periferia, también asociado al de desarrollo polarizado o desarrollo desigual, aparece ineludiblemente en nuestros países y se replica también al interior de las regiones, lo que explicado en términos de Polèse (1998), tiene que ver con "el impacto acumulativo de los movimientos en los factores de producción (trabajo, capital, conocimientos, etc.) en beneficio de las regiones que fueron las primeras en desarrollarse, y en detrimento de las regiones alejadas, acaba a menudo creando lo que se ha acordado en llamar una relación centro-periferia." (p. 203). 


\section{La gobernanza urbana}

El proceso urbano es más un aspecto pasivo que activo dentro del desarrollo políticoeconómico. La urbanización es un proceso social de base espacial donde una amplia gama de actores diferentes, con objetivos completamente distintos, se interrelacionan mediante una configuración determinada de prácticas espaciales entrelazadas. La gobernanza urbana va mucho más allá del gobierno urbano: el poder de organizar la ciudad está en su gobernanza, entendida como una coalición más amplia de fuerzas dentro de las cuales el gobierno y la administración urbana sólo desempeñan una función facilitadora y de coordinación.

En el ámbito de las prácticas espaciales aparecen nuevos patrones y estructuras ecológicas dentro de la nueva forma urbana, denominada como ciudad extendida, ciudad difusa o ciudad región. Estos son importantes para entender su implicación en el modo de organizar la producción, el intercambio y el consumo; fundamentales para establecer las relaciones sociales de ejercer el poder tanto económico como político y para alcanzar la integración espacial de la acción social. La planificación territorial tendrá, en este sentido, que ir más allá de las tajantes divisiones tradicionales de área urbana independiente del área rural, o de los estrechos límites municipales para entender que se traslapan y se complementan.

\section{El cambio al empresarialismo en la gobernanza urbana}

La erosión de la base económica y presupuestal de muchas ciudades del mundo capitalista, del que no se escapan nuestras ciudades, obliga a que los gobiernos urbanos sean mucho más innovadores y emprendedores para aliviar sus estrecheces. Este cambio al empresarialismo lo han implementado gobiernos urbanos de distintas tendencias políticas, con bases jurídicas y apuestas políticas diferentes, con el que pretenden enfrentar las consecuencias negativas de la globalización, como la desindustrialización y el desempleo generalizado. Ya no es el Estado-nación el que controla los flujos multinacionales; la inversión se da como una negociación entre el capital financiero internacional y poderes locales que hacen lo posible por maximizar el atractivo del espacio local.

Harvey (2007b) plantea que las evidencias empíricas obtenidas frente a cómo se dan las estrategias de alianzas y coaliciones para influir en la promoción local demuestran que:

- El nuevo empresarialismo tiene, como elemento central, la alianza del sector público con el sector privado, se integra el uso de los poderes gubernamentales locales para atraer la financiación externa.

- La actividad que se desprende de esta alianza público-privada es empresarial, su ejecución y diseño son de naturaleza especulativos, ya que el sector público asume el riesgo y el sector privado obtiene los beneficios.

De igual manera considera las siguientes opciones básicas como estrategias de empresarialismo urbano, que antes de ser excluyentes son complementarias:

- La competencia dentro de la división internacional del trabajo supone la posibilidad de explotación de determinadas ventajas para la producción de mercancías y servicios.

- La región urbana puede intentar mejorar su posición competitiva con respecto a la división espacial del consumo. El estilo consumista de la urbanización promueve una base cada vez más amplia para la participación en el consumo de masas, donde se incluyen el negocio turístico y los acontecimientos culturales para captar actividades de inversión.

- El empresarialismo ha estado fuertemente marcado por una lucha feroz por la adquisición de funciones clave de control y mando en las altas finanzas, en el gobierno, y en el procesado de información, incluidos los medios de comunicación.

El filo competitivo, con respecto a las redistribuciones de excedentes mediante la administración central, sigue siendo de importancia suma, y en ella, las alianzas de 
clase dominante urbana tienen más oportunidades de explotarlos.

En síntesis, la tarea de la gobernanza urbana consiste en atraer a su espacio unos flujos altamente móviles y flexibles de producción, financiación y consumo.

\section{Políticas regionales o políticas de desarrollo regional}

Los desajustes particulares al interior de las regiones, o problemas específicos, de carácter estructural y/o coyuntural pueden justificar la intervención del Estado en la economía regional, como en otros aspectos de la vida humana, sobre todo, cuando considera que los resultados del mercado son inaceptables o inferiores al óptimo social deseable. Se habla entonces que el Estado entra a contrarrestar las fallas del mercado en el ámbito regional, las que suelen estar motivadas por un deseo de alcanzar una mayor equidad.

Son dos las grandes categorías de política económica de carácter regional las que Polèse (1998) nos define; estas, en la práctica, antes que excluirse se mezclan y se complementan:

Las políticas regionales, propiamente, como políticas económicas de redistribución de la actividad económica dentro del espacio nacional, tienen como objetivo resolver las disparidades regionales, y su competencia es a nivel nacional. El Estado central puede subvencionar a particulares o a gobiernos locales con el objetivo de reducir desigualdades mediante un régimen de impuestos progresivo, transferencia a las personas: seguro de desempleo, prestaciones a familias con hijos, educación y salud pública de alta calidad.

Las políticas de desarrollo regional, fomentadas desde las actuales exigencias de descentralización de la economía globalizada, son de carácter particular, tienen como objetivo resolver la inequidad regional dentro de un territorio específico. Como políticas de redistribución de los ingresos entre las poblaciones regionales, son de competencia de su escala regional o local, y, desde el potencial del medio, deben aprovechar sus recursos locales, revalorizarlos con procesos manufactureros apropiados, potencializando a su vez las redes de interacción y solidaridad de sus comunidades.

\section{Reposicionamiento de las ciudades y las regiones en la economía global}

En la forma específica de relación entre sociedad y territorio, evidenciada por la interrelación de tres macroprocesos: la globalización, la informacionalización y la difusión urbana generalizada; la fuerza de la tendencia apunta hacia la desaparición de la ciudad. Pero en contravía de esta visión, Borja y Castells (2004) plantean la necesidad de renovar el papel específico de las ciudades en un mundo de urbanización generalizada, proponiendo la construcción de una relación dinámica, creativa y particular entre lo local y lo global, sustentado en la posibilidad de un control de los ciudadanos sobre su propia vida.

Frente al reto que plantea el fenómeno de la globalización, las ciudades, en cuanto a sus políticas urbanas, tendrán que ir más allá de las preocupaciones familiares de resolver sus problemas urbanos cotidianos. Deben asumir las consecuencias que implican la globalización, la de asumir políticas de desarrollo regional para construir una dimensión urbana más fuerte, que no siendo específicamente urbana, tienen agudos impactos urbanos.

Frente a los temas críticos que plantea la globalización a la gobernanza urbana, Sassen (2007) nos muestra que estos se manifiestan dentro de dos procesos principales: i) cambios en las escalas, espacios y contenidos de la actividad económica que se manifiesta en la conformación de ciudades y regiones urbanas globales con tendencia a la conformación de mega-regiones, y la conformación de flujos transfronterizos, y ii) ajuste a la nueva tendencia para maximizar sus beneficios, que para las ciudades implica cambios en las interpretaciones y esquemas políticos, con desafíos que van desde lo económico a lo social. 
Este nuevo espacio geográfico, de escalas urbanas crecientemente grandes, donde ciudades y áreas metropolitanas se mezclan entre sí, conduce también a infraestructuras interregionales como transporte y servicios públicos, de donde emerge un territorio diverso a su interior, que requiere sus propias formas de planificación y coordinación regional, las que deben ser exploradas con nuevas estrategias de desarrollo que permitan generar ventajas, tanto para las áreas más avanzadas como para las menos avanzadas, dentro de la mega-región.

La escala territorial de una región es más real en su representación de un corte transversal de las actividades económicas de un país, que la escala local de la ciudad. Por ejemplo, es probable que incluya como variables clave su producción, su manufactura y su conjunto de actividades económicas asociadas y ubicadas en un polo estratégico de la economía nacional, que no solo incluyen a la ciudad, sino toda su área de influencia inmediata, la ciudad-región.

En lo que respecta a los beneficios de crecimiento resultante de la globalización, puede decirse que la ciudad-región global permite ver las posibilidades de un tipo de crecimiento más distribuido, una extensión más amplia de sus beneficios. "Una de las ventajas de la densidad urbana central es que ha ayudado históricamente a resolver el riesgo de variedad insuficiente. Trae consigo mercados de trabajo diversos, redes de firmas y colegas diversas, enormes concentraciones de tipos diversos de información sobre los últimos desarrollos." (Sassen, 2007, p. 25).

Para nuestras ciudades intermedias es importante entender que pueden formar parte de una región, o que la sumatoria de ellas también puede conformar una región, ya que para la actual economía global, si bien la centralidad sigue siendo un aspecto clave, hoy en día, ya no existe una relación directa única entre centralidad y entidades geográficas tales como el centro de la ciudad, o el distrito central de negocios. Estas nuevas relaciones espaciales tendrán implicaciones territoriales importantes que van a incidir notoriamente en las políticas urbanas y la planificación territorial.

\section{Los retos de la política urbana}

Frente a los retos de política urbana que tienen que enfrentar nuestras naciones para contrarrestar los efectos negativos de la globalización, se plantea asegurar una mayor convergencia territorial que se oponga a la excesiva focalización que demanda la competitividad en la economía globalizada.

Una ciudad o región puede construir fortalezas en términos de sus "diferencias especializadas", sobre las especificidades de su historia económica, en orden de alcanzar una diferenciación especializada para desarrollar su economía del conocimiento. Estos tipos de diferencias se vuelven, por tanto, importantes para entender las nuevas estrategias que debe asumir una ciudad o una región, que como nos lo explica Sassen (2007) está en función de dos razones: la primera, desde el giro de una economía espacial keynesiana que busca la convergencia territorial nacional, a una economía espacial post-keynesiana orientada hacia la focalización territorial y, la segunda, la ventaja de la ciudad-región en la economía global, en función del posicionamiento en circuitos muy especializados.

En lo que respecta a las implicaciones entre la planificación del desarrollo urbano regional y las exigencias de la economía del conocimiento, se hace necesario desarrollar entendimientos más amplios y completos de sus parámetros y condicionalidades. "Si la relación entre la antigua economía material (manufactura, minería, agricultura, etc.) y la actual economía del conocimiento es repensada, es posible ver que esto no es un conflicto como típicamente se piensa, sino que la anterior puede alimentar a la primera." (Sassen, 2007, p. 33).

\section{De lo local a lo global}

La perspectiva de desarrollo local surge en la década de 1980 como reacción al proceso de globalización y ante la insuficiencia de las políticas macroeconómicas de desarrollo, para resolver problemas asociados con la creación del empleo y la mejora del bienestar social, y se centra en darle una mayor presencia a los niveles locales y regionales en la planificación del desarrollo. 
Las dos tendencias, globalización y localización, se tornan en las dos caras de una misma moneda, la una se necesita de la otra. Entender esta disyuntiva y obtener lineamientos que contribuyan a alimentar criterios para comprender cuál debe ser la respuesta desde las regiones para encaminarse por la senda de un desarrollo sustentable y a escala humana, es el reto que anima a la reconceptualización de muchas disciplinas en las que su objeto de estudio se relaciona con el fenómeno de la espacialidad.

Autores como Boisier (2005) hacen énfasis en que en el actual contexto de la globalización, el territorio desempeña un papel más importante que en el pasado. La globalización estimula el surgimiento de procesos de crecimiento local, pero esto no significa que necesariamente estimule también procesos de desarrollo local, por su naturaleza inequitativa en la distribución de las ganancias.

La complementación que se plantea entre lo global y lo local, como una alternativa que abra la puerta a la posibilidad de la sobrevivencia de las culturas basadas en lo local, sugiere una atención conjunta de la localización de lo global y de la globalización de lo local. La presencia de estos dos ámbitos espaciales relacionándolos en un mismo campo la reafirma Boisier (2005) en la siguiente referencia: "La síntesis se encuentra más bien en el neologismo de Robertson, el término "glocal": piensa global y actúa local (para la empresa) y piensa local y actúa global (para el territorio)" (p. 51).

Una sociedad y su contexto urbano-regional pueden considerarse como glocal en la medida en que, dentro de su dinámica, no niegue sus características culturales, históricas y sociales propias, a la vez que responde a las exigencias de una economía globalizada, por tanto, ser glocal implica relacionarse con el mundo con conocimientos únicos que entrarían en diálogo con lo externo. En este sentido, el desarrollo local dentro de la globalización es una resultante directa de la capacidad de los actores y de la sociedad local para estructurarse y movilizarse, con base en sus potencialidades y su matriz cultural, para definir y explorar sus prioridades y especificidades.

En un importante trabajo de PNUD/OIT/UNOPS/EUR (2002) se destacan algunos puntos relevantes sobre el desarrollo local: i) el desarrollo de un territorio está fuertemente condicionado por la voluntad y capacidad de los actores locales; ii) el desarrollo de un territorio gira alrededor de la valorización de las potencialidades locales; iii) en todas partes se ha comprobado la importancia de la pequeña y mediana empresa; iv) el desarrollo depende de la capacidad de integrar las iniciativas empresariales; v) el territorio debe dotarse de instrumentos adecuados, y vi) el secreto del éxito reside en la capacidad de interacción activa entre lo local, lo nacional y lo internacional.

Avanzar en este proceso de conceptualización redundará en tener mayores elementos y claridad de criterios con que se puedan orientar las apuestas por el ordenamiento y uso del suelo del lugar, sin descuidar ninguna de las dimensiones que en ella intervienen. El 'conocimiento local' es, en sí, el elemento fundamental para el desarrollo de la identidad regional, vital para las relaciones de la gente con los entornos no humanos y para la existencia y estructuración de modelos culturales de la naturaleza.

\section{Gestión del riesgo y globalización}

\section{La Gestión del riesgo}

La planificación, el monitoreo y la evaluación de la Gestión del riesgo (GdR) son algo novedosos; aparecen en la escena del debate nacional e internacional con posterioridad al huracán Mitch en Centroamérica, a finales de los 90, de donde emergió el planteamiento de anticipar riesgos hacia el futuro y, por tanto, intervenir en los mecanismos de planificación y de formulación de los proyectos de inversión tomando en cuenta zonas y grados de inseguridad. La GdR se expresa ya en una doble intervención: correctiva y prospectiva.

El Marco de Acción de Hyogo (MAH), producto de la Conferencia Mundial sobre Desastres, convenido por las Naciones Unidas y celebrado en Kobe, Japón, en 2005, se ha convertido en el referente internacional más conocido en torno a los objetivos de la GdR. Las prioridades centrales del MAH se refieren a aspectos de gobernabilidad, conocimiento, educación y cultura, las causas subyacentes del riesgo, y los preparativos y respuesta a desastres. 
La gestión integral del riesgo es un proceso concatenado de análisis de amenazas, conocimiento de las vulnerabilidades, que al actuar en manera prospectiva implica la prevención mediante la disminución de las amenazas y mitigación mediante la intervención en la vulnerabilidad. Actuar de manera reactiva implica la atención de las emergencias, rehabilitación y reconstrucción de zonas de desastre. Este proceso tiene como gran objetivo romper el círculo cotidiano de desastres - reconstrucción escenarios de desastres.

Una nueva definición, donde aparece la cultura como elemento central, es elaborada por Wilches-Chaux (2001): "La gestión del riesgo es el instrumento que nos aporta la cultura para que manejemos adecuadamente nuestra relación con las amenazas del medio, al igual que nuestras debilidades o vulnerabilidades frente a esas amenazas, de manera tal que los riesgos que surgen de la confluencia de las dos, no necesariamente se convierta en desastres." (p. 6).

\section{Gestión del riesgo en el contexto de la globalización}

Al disponer el Estado de menos recursos en función del bienestar de sus pobladores, como consecuencia de la desregulación, la privatización y el abandono del sistema de provisión social, conlleva la reducción de su papel asistencialista a nivel de educación, salud y servicios sociales y se impone la determinación neoliberal de transferir nuevamente al individuo toda la responsabilidad para su bienestar, incluido el asumir todo tipo de riesgos. Es así como la incapacidad personal se atribuye por regla general a fracasos individuales. Esta misma lógica también impera en materia de gestión del riesgo, pretende que sean los individuos, los que asuman todos los riesgos; y aquí, por ejemplo, aparecen los mercados de seguros con sus empresas (naturalmente de objeto comercial), que entran a actuar con el objeto de proteger al ciudadano, para terminar lucrándose de la seguridad como una mercancía más.

Uno de los elementos que prefiere la gobernanza urbana, en su relación directa con el empresarialismo, es la mercantilización del suelo. Especular con el uso del suelo urbano en las zonas céntricas y bien ubicadas de la ciudad es uno de los principios que mueve el mercado inmobiliario. ¿Cómo concertar ahora todos estos intereses vitales en la gestión del suelo urbano, cuando estos se ven potencialmente en un alto riesgo, cuando están ubicados en una zona considerada como de amenaza alta originada por fenómenos naturales?, o desde otra perspectiva extrema, ¿Será esta la oportunidad para que estos suelos y sus construcciones se devalúen, se deterioren, y ahí sí, hacer la gran apuesta, adquirirlos a precio de ruina para esperar que, frente a nuevos desarrollos tecnológicos, o nuevas políticas de aseguramiento y consideración del riesgo, permitan especular con estos inmuebles?

Para garantizar la movilidad del capital entre regiones y países, la globalización económica exige de los países que las barreras como "La planificación y los controles medioambientales, así como otros impedimentos localizados, han de ser eliminadas, salvo en aquellas áreas que son cruciales para los intereses nacionales" (Harvey, 2007a, p. 73). La alternativa que tiene el Estado de intervenir frente a lo relativo a las fallas del mercado, donde las empresas no asumen todos los costos imputados a su actividad, como los de la contaminación, los neoliberales se muestran profundamente desconfiados hacia este tipo de intervenciones. Pero, dentro de la lógica de una política regional territorial, al servicio del capital, el manejo de los riesgos juega un papel importante en la competitividad de una ciudad. Por tanto, una estrategia de gobernanza urbana para mejorar su entorno espacial, sería garantizar, o dar condiciones para la minimización del riesgo, ante todo, cuando de protección de vidas humanas se trata.

\section{Integración de dimensiones de gestión del riesgo, ambiental y adaptación al cambio climático}

En el mundo hoy en día, ante la evidencia del sinnúmero de desastres asociados a las amenazas de los fenómenos naturales, se produce una creciente promoción y apoyo a la llamada "adaptación" al cambio climático (ACC), tema de creciente envergadura a nivel de organismos internacionales que apoyan y presionan a los países para avanzar 
en la mitigación y prevención de los riesgos de desastre. En particular, a partir del Panel Intergubernamental para el Cambio Climático (IPCC, por sus siglas en inglés), con el apoyo de la Estrategia Internacional de las Naciones Unidas para la Reducción de Desastres (EIRD) y la constitución, en 1999, de la oficina de Naciones Unidas para la Reducción del Riesgo de Desastres UNISDR.

En el marco de la EIRD, el estudio elaborado en 2009 bajo el título "Pobreza y Riesgo en un Clima Cambiante", en sus conclusiones arrojan que es a través de mejoras en el manejo ambiental, el ordenamiento territorial, la sostenibilidad de los medios de vida y la gobernanza que se logrará apoyar simultáneamente la reducción del riesgo de desastre, la reducción de la pobreza y el fomento de la adaptación y mitigación al cambio climático.

Autores como Lavell (2010), Lampis (2014), Wilches-Chaux (2001), entre otros, nos hablan de la importancia y conveniencia de la integración sinérgica de estas tres dimensiones: la Gestión Ambiental (GA), la Gestión del Riesgo de Desastre (GRD) y el contexto del cambio climático (CC), para los procesos de inversión pública y planificación a nivel regional y mundial. Es el esfuerzo que se plantea como el nuevo paradigma a incluir para el desarrollo de las políticas públicas; así hayan surgido cada una individualmente, con sentidos propios, y objetivos específicos particulares, pero integrados frente a un gran objetivo de orden superior: el desarrollo sostenible.

\section{Espacios para la inclusión de la gestión del riesgo en la planificación territorial}

La planificación territorial y su concreción en el ordenamiento territorial es la capacidad para determinar qué se puede hacer y qué no se puede hacer en cada porción del territorio o región. Es un conjunto de decisiones políticas y técnicas que deben tomarse de manera participativa, con la intervención de todos los actores y sectores sociales. Al respecto, Lavell (2010b) insiste en que "El territorio se construye por la interacción de procesos sociales con los recursos del ambiente natural, incluyendo el recurso de localización. La seguridad territorial se construye con referencia a los medios de vida y sus soportes, y a través de la utilización de nociones de uso máximo y los planes de uso del suelo" (p.16).

Frente a los riesgos de desastres de origen natural, el ordenamiento y la planificación del territorio tienen como objetivo claro el de mitigar los riesgos que afrontan las comunidades ya asentadas, y el de no dar lugar a nuevos riesgos en la futuras áreas de expansión. Por tanto, en la actualidad y como prioridad, la planificación y la inversión pública y privada tendrán que abordar en forma transversal las nuevas variables de la GdR y la GA frente a la adaptación al cambio climático, si quieren apostarle a ir por la senda del desarrollo sostenible.

A la planificación territorial se le abre un gran espacio en el reconocimiento, que desde instituciones internacionales se tiene, de que el riesgo de desastre, la degradación ambiental, y el proceso de adaptación al cambio climático, además de los procesos y medios de vida de la población, tienen un claro referente territorial, y sus expresiones más concretas remiten al nivel local o micro territorial. Al respecto, Lavell (2010b) nos introduce un elemento complementario: "la noción de la "seguridad territorial" permite captar un objetivo fundamental, central y sinérgico de las tres gestiones. Por seguridad territorial se entiende la existencia de condiciones, tanto sociales como ambientales, que sostienen la existencia segura de medios y proceso de vida en distintas circunscripciones territoriales o regionales" (p. 45).

Las ciudades tienen un papel esencial en garantizar el desarrollo sostenible. Las ciudades, por su naturaleza de concentrar grandes densidades poblacionales, son las mayores consumidoras de recursos naturales y las mayores productoras de residuos que contribuyen en gran medida a los problemas medioambientales, pero también son parte de la solución de ellos. Las ciudades tienen una gran capacidad de actuar a favor de un desarrollo sostenible, condición necesaria para su supervivencia. 
La gestión del riesgo queda implícita dentro, tanto del propósito de la calidad de vida como en el de la reproducción de las condiciones materiales; vivir en constante riesgo afecta la calidad de vida. Habitar una zona donde se es vulnerable a una amenaza no garantiza condiciones para un desarrollo sostenible, por tanto, la organización del espacio urbano debe tener condiciones adecuadas para garantizar la habitabilidad de sus habitantes.

Como conclusión, se deben cambiar nuevamente las reglas del juego, y estas deben incluir medidas para sosegar la globalización. Contrarrestar las nefastas consecuencias de la globalización en nuestros países y regiones periféricas sólo puede hacerse con la vigilancia ciudadana y una democracia ecológica local que cambie el poder de los negocios centralizados por el poder de hacer las cosas, y de proteger el equilibrio irrigando recursos a la comunidad.

\section{Conclusiones}

\section{Desde la Globalización}

Ante la proliferación, de una manera tan generalizada a escala mundial del modelo político-económico neoliberal, reconocer su historia desde una perspectiva crítica sirve para proponer un marco base para identificar y construir acuerdos políticos y económicos alternativos, sobre todo desde nuestras regiones latinoamericanas que han sido las perdedoras en la implantación de este modelo.

La competencia de los Estados y aún más de sus regiones, por la ubicación de actividad económica global o su inserción en los mercados globales, tiene muchos bemoles para su análisis. Por un lado están las ventajas económicas que traerían para el territorio, pero en esta economía global neoliberal, para que se den países ganadores, siempre habrá países perdedores. Igual que al interior de los países, a unas regiones llegarán las mieles del desarrollo y a otras sus devastadoras consecuencias. Similar para el nivel local, unos serán los sectores beneficiados y otros los perjudicados, los que acarrean con la pobreza como consecuencia de un subempleo pésimamente remunerado debido a las altas tasas de desempleo, característica de este actual modelo neoliberal.

Se podría afirmar que, para probar la hipótesis que trata de explicar por qué en nuestros países en desarrollo no hemos avanzado en la gestión del riesgo, sobre todo en lo que se relaciona con amenazas de origen natural, obedece precisamente a la falta de voluntad política para llevarlo a cabo en la práctica con políticas regionales. Ya que en función de los postulados que proponen las teorías neoliberales que han asumido dócilmente nuestros Estados, el manejo del riesgo pasa a ser del dominio de la responsabilidad individual, más que como una política de Estado que contribuya a su eliminación o minimización.

Tras la disolución del Estado de Bienestar, el Estado tiende al máximo, a deshacerse de sus responsabilidades de hacer grandes inversiones públicas en materia de gestión del riesgo que le apunten a prevenir y minimizar el riesgo. Investigar a fondo la amenaza y fomentar la apropiación social de este conocimiento por parte de las comunidades afectadas y, sobre todo, apostarle principalmente a la minimización del riesgo, muchas veces exigen la construcción de costosas infraestructuras para mejorar las condiciones de habitabilidad de las zonas vulnerables. Por otro lado, la presencia de riesgos no mitigables requerirá habilitar nuevas áreas seguras para estas poblaciones vulnerables. Alternativas todas estas que si se dejan a la libre oferta del mercado, tal como lo pregona el modelo neoliberal, nunca aparecerán.

Uno de los desafíos a resolver, que exige la globalización a las regiones para ser competitivas, es el de la seguridad, y esta va más allá de la sola seguridad ciudadana, se extiende a lo que se ha denominado la seguridad territorial, donde encuentran espacio la gestión del riesgo y la gestión ambiental como garantes de la habitabilidad del territorio. A la par de este requerimiento, a nivel global, también son cada vez más relevantes los del desarrollo sostenible. Y la sostenibilidad como un desafío del territorio ante la globalización debe responder a las exigencias de competitividad y calidad de vida. Desde esta óptica se debe buscar que no haya contradicción insuperable entre 
competitividad e integración social, entre crecimiento y calidad de vida, ya que las ciudades más competitivas internacionalmente son aquellas que ofrecen mayor calidad de vida a sus habitantes.

Frente a esa necesidad latente, que nuestros países en desarrollo tienen para actuar inmediatamente para contrarrestar los efectos desastrosos del cambio climático y demás situaciones de vulnerabilidad ante los riesgos de desastres, en aras de una radicalización política, no se puede esperar hasta que se den condiciones estructurales que cambien el actual sistema dominante, para actuar efectivamente en esta dirección. Siendo pragmáticos, desde las iniciativas locales y con el compromiso de sus actores locales, las ciudades y sus regiones deben encontrar oportunidades en el actual contexto de la globalización que les permita acceder a recursos que financien los programas y proyectos que se requieran.

Dentro de las oportunidades de la globalización económica que hoy encuentran nuestros países en desarrollo, pueden presentarse los compromisos que adquieren nuestros Estados con los organismos internacionales, donde hay requerimientos para que se den las condiciones de reproducción del capital, y se exige también brindar condiciones de vida y seguridad para los actores de la economía. Este, por ejemplo, sería el caso colombiano, cuyo gobierno está tramitando su ingreso a la Organización de Cooperación y Desarrollo Económico OCDE.

Desde esta perspectiva existen programas mundiales y organismos internacionales, que brindan líneas de apoyo y créditos para acceder a recursos que vayan en esta dirección. Por ejemplo, las Acciones para la Adaptación al Cambio Climático recomendadas a los países desde Naciones Unidas, es el espacio donde la gestión del riesgo y su inclusión en la planificación territorial pueden encontrar la oportunidad para acceder a recursos para concretar sus propósitos.

\section{Desde la planificación del territorio}

En particular, frente a la gestión del riesgo, Estados de constituciones garantistas -como se pregonan nuestras naciones- deben ante todo garantizar la vida y bienes de sus ciudadanos. En temas como los de la seguridad territorial, sobre todo, en los que permitan minimizar el riesgo por amenazas de origen natural, es el Estado el primer actor que debe intervenir y regular con sus instituciones y sus políticas regionales. El Estado, en su manifestación de gobierno local, es quien tendrá como responsabilidad incluir la gestión del riesgo como una variable importante a tener en cuenta en la planificación territorial regional, y regular así el uso y la ocupación del suelo, en función de evitar al máximo que sus pobladores estén expuestos a las amenazas de tipo natural.

La escala regional es la pieza clave que tienen los Estados para la ordenación sostenible de sus territorios. La elaboración de planes regionales de ordenamiento general y sectorial, otorgan protagonismo destacado a la reducción del riesgo. Incluir dentro de la planificación territorial regional la gestión del riesgo, se constituye en una estrategia para el desarrollo local, y una alternativa para responder a los retos de competitividad que plantea la globalización para las regiones dentro de un país.

Entre los distintos espacios desde donde puede ser posible encontrar senderos que contribuyan a la inclusión de la gestión del riesgo en la planificación territorial, y que son fomentadas desde las actuales exigencias de descentralización de la economía globalizada, se destacaría la constitución de la ciudad región como nueva alternativa de planificación para encontrar espacios competitivos en los mercados de la globalización. Pero, si se trata de gestionar el riesgo como una variable directriz de la planeación territorial regional, deberá acudir a la formulación de políticas de desarrollo regional, obviamente promovidas y lideradas desde sus autores locales, ya que desde la políticas de globalización, las políticas regionales nacionales no están interesadas en intervenir en zonas marginales y periféricas donde se asocian las áreas más deprimidas económicamente con la más vulnerables a riesgos relacionados con amenazas de origen natural. 


\section{Desde el Desarrollo Local}

Cómo responden las regiones a las exigencias de doble vía global-local, es la disyuntiva que, desde las posiciones derrotistas, consideran que no hay nada que hacer frente al fenómeno de la globalización, o desde la negacionista, que hace oídos sordos al mismo. Pero, desde el desarrollo local se asume una visión pragmática, que sin desconocerlo, intenta sacarle provecho. Desde esta perspectiva, al considerar que ya la globalización está aquí, lo mejor que se puede hacer, es convertirla en una oportunidad para el desarrollo local, que permita obtener un mayor acceso a recursos globales disponibles.

En definitiva, el desarrollo local en el contexto actual de globalización puede suavizar los efectos perversos de un proceso dominante realizado de forma poco democrática y sin contar para nada con la opinión de las personas y las particularidades culturales de las sociedades. Al mismo tiempo, puede incluso decidir cómo quiere realizar ese desarrollo, lo que supondría participación democrática y no quedar expuestos, sin posibilidad alguna de actuación, a las decisiones que sobre "el mundo" toman desde los despachos, con visiones centradas exclusivamente en el beneficio económico para unos pocos y en el perjuicio para muchos.

Para avanzar en esa necesaria integración de las dimensiones ambientales y de gestión del riesgo en el actual contexto de la adaptación al cambio climático como elementos transversales de la planificación del territorio, se requiere de visiones holísticas, integrales e integradas, que trascienden la esfera de acción limitada o parcializada que proveen distintas disciplinas científicas o prácticas profesionales particulares con su visión, a veces demasiado fraccionada y especializada del mundo. Este es un espacio que desde las universidades regionales, como las más conocedoras de sus respectivos contextos locales hagan sus grandes aportes.

En síntesis, hablar de desarrollo regional centrado en los recursos y en las capacidades locales, implica un modo de intervención política que ponga énfasis en el medio como fuente de desarrollo económico impulsado desde la base, que se sustenta en las empresas y redes locales de colaboración y solidaridad, que incorpore la dimensión social y una preocupación por las particularidades locales.

\section{Referencias bibliográficas:}

Alcañiz, M. (2008). El desarrollo local en el contexto de la globalización. Convergencia, (47), 285-315. Recuperado de http://www.redalyc.org/articulo.oa?id=10504711

Boisier, S. (2005). ¿Hay espacio para el Desarrollo local en la globalización?. Revista de la CEPAL, (86), 47-62.

Borja, J. \& Castells, M. (1997). Local y global. La gestión de las ciudades en la era de la información. Barcelona: Taurus.

Brand, P. (2009). La ciudad latinoamericana en elsiglo XXI: Globalización, Neoliberalismo, Planeación. Medellín: Universidad Nacional de Colombia.

De Mattos, C. (2010). Globalización y metamorfosis urbana en América Latina. Quito: Organización Latinoamericana y del Caribe de Centros Históricos (OLACCHI).

Escobar, A. (2000). El lugar de la naturaleza y la naturaleza del lugar: ¿Globalización o postdesarrollo?. Carolina del Norte: Universidad North Carolina.

Fondo para la Reconstrucción y Desarrollo Social del Eje Cafetero - FOREC (2001).

Zonificación de amenazas. Elementos conceptuales y metodológicos de la gestión del riesgo a la planeación y el ordenamiento territorial. Serie: Ordenamiento Territorial y Reconstrucción Eje Cafetero - Vol. VII. Bogotá, Colombia.

Harvey, D. (2007a). Breve historia del neoliberalismo. México: Akal.

Harvey, D. (2007b). Espacios del capital. Hacia una geografía crítica. México: Akal.

Lampis, A. (2014). Ciudad y resiliencia: una cuestión al estilo de Escher. Territorios, (28), 9-19 
Lavell, A. (2010a). Gestión Ambiental y Gestión del Riesgo de Desastre en el contexto del Cambio Climático: Una aproximación al desarrollo de un concepto y definición integral para dirigir la intervención a través de un Plan Nacional de Desarrollo. Bogotá: Departamento Nacional de Planificación - DNP.

Lavell, A., \& otros. (2010b). Lecciones Aprendidas de la Gestión del Riesgo en Procesos de Planificación e Inversión para el Desarrollo. Taller Internacional Riesgo y Cambio Climático. Lima. Recuperado de: http://www.unisdr.org/files/18953_ leccionesderiesgovf911.pdf

Max-Neef, M. (1994). Desarrollo a escala humana. Conceptos, aplicaciones y algunas reflexiones. Barcelona: Icaria.

OCDE (Organización de Cooperación y Desarrollo Económicos) (2001): Devolution and Globalisation. Implications for Local Decision-Makers. París.

PNUD/OIT/UNOPS/EUR (Programa de las Naciones Unidas para el Desarrollo/ Organización Internacional del Trabajo/Oficina de las Naciones Unidas de Servicios para Proyectos/EUR). (2002).

Polèse, M. (1998). Economía urbana y regional. Costa Rica: Libro Universitario.

Sassen, S. (2007). El reposicionamiento de las ciudades y regiones urbanas en una economía global:Ampliando las opciones de políticas y gobernanza. Eure, 33(100), 9-34. Recuperado de http://doi.org/10.4067/S0250-71612007000300002

Sen, A. (2000). Desarrollo y libertad. Barcelona: Planeta.

Stiglitz, J. (2002). El malestar en la globalización. Barcelona: Círculo de Lectores.

Wilches-Chaux, G. (2001). Herramientas sociales para la gestión del riesgo. Bogotá: FOREC 


\title{
"Mall"-deando la ciudad: 24 años de territorialización de los malls en Costa Rica
}

\author{
Andrés Jiménez Corrales
}

Invitado nacional

Universidad de Costa Rica, Costa Rica

ajimenez2812@gmail.com

Recibido: 17 de mayo 2017

Aprobado: 4 de julio 2017

\section{Andrés Jiménez Corrales}

Licenciado en Geografía por la Universidad de Costa Rica. Estudiante en la Maestría Académica en Geografía en la Universidad de Costa Rica. Actualmente trabaja como investigador y docente en la misma universidad. Entre sus intereses de investigación se encuentran: producción del espacio, territorio y procesos de territorialización, urbanismo neoliberal y disparidades urbanas.

\begin{abstract}
Resumen:
La territorialización de los malls en Costa Rica inicia en el año 1993 con la inauguración de Multiplaza Escazú, en la Gran Área Metropolitana de Costa Rica. De este año al año 2017 se contabiliza un total de 14 malls. En este artículo se hará un recorrido sobre las principales características que tienen los malls; se caracterizará la llegada del primer mall al país y se desarrollará un análisis de la evolución espacio-temporal que han tenido estas infraestructuras en la GAM. Por último, se hace un repaso de algunos de los reclamos y respuestas ciudadanas que se han dado ante la construcción de un mall.
\end{abstract}

Palabras clave: mall; centro comercial; territorialización; urbanismo neoliberal.

\section{"Mall-ding" the city: 24 years of mall territorialization in Costa Rica}

\begin{abstract}
:
The territorialisation of malls in Costa Rica began in 1993 with the inauguration of Multiplaza Escazú in the Greater Metropolitan Area of Costa Rica. From 1993 to 2017 a total of 14 malls were built. In this article, a route across the main characteristics of malls is made; the arrival of the first mall to the country is characterized and an analysis of the space-time evolution that these infrastructures have had in the GAM is developed. Finally some of the complaints and citizen responses that have been given to the construction of a mall are reviewed.
\end{abstract}

Keywords: mall; shopping center; territorialization; neoliberal urbanism. 